\title{
Schweiz wieder im zweiten und dritten Rang
}

2004 hatte die Schweiz gemessen am Anteil des Bruttoinlandsprodukts wie im Vorjahr die zweithöchsten Gesundheitsausgaben der Welt. Unverändert blieb auch der dritte Rang bezüglich Pro-Kopf-Ausgaben und der extrem hohe Selbstzahleranteil. Die Spitalbettendichte ist durchschnittlich, die Medizingerätedichte sehr hoch.

G. Kocher

Literatur

1 OECD Health Data - Statistics and Indicators for 30 Countries. Paris: OECD; 2006. ISBN 92-64-02279-1. Sechssprachig, mit Benützerhandbuch. Die Einzelplatzversion kostet $€ 80$ (im Vorjahr noch über $€ 200$ !). Einzelheiten bei www. oecd.org/health/healthdata. Im Spätherbst publiziert die OECD das Buch «Health at a Glance OECD Indicators 2006», das einen Teil der Statistiken sowie Erläuterungen und Grafiken enthalten wird.

Korrespondenz:

Dr. rer. pol. Gerhard Kocher

Haldenweg $10 \mathrm{~A}$

CH-3074 Muri b. Bern

Tel. 0319526707

kocher@spectraweb.ch
Im Juni erscheint jeweils der neueste repräsentative Überblick über das Gesundheitswesen der OECD-Länder: die CD «OECD Health Data Statistics and Indicators for 30 Countries» [1]. Die diesjährige Jubiläumsausgabe (15 Jahre) enthält Daten zu Gesundheitszustand, Ressourcen, Inanspruchnahme, Gesundheitsausgaben, Finanzierung, sozialem Schutz, Arzneimittelmarkt, nichtmedizinischen Bestimmungsfaktoren des Gesundheitszustandes sowie demographische und wirtschaftliche Kennziffern. Insgesamt sind es über 1200 Indikatoren. Die meisten Zeitreihen enthalten Daten bis zum Jahr 2004, andere nur bis 2003. Viele gehen zurück bis auf 1960

\section{Anteil der Gesundheitsausgaben am BIP}

Gegenüber dem Vorjahr ist 2004 der Anteil der nationalen Gesundheitsausgaben am Bruttoinlandsprodukt in 14 von 25 Ländern gestiegen. In fünf Ländern blieb der Anteil am BIP unverändert, in sechs Ländern sank er (für die anderen fünf OECD-Länder liegen die Zahlen für 2004 noch nicht vor). In der Schweiz stieg er nur leicht von 11,5 auf $11,6 \%$, weil in diesem Jahr unser BIP kräftig zunahm (um 2,6\%).

Nach den USA mit ihren extrem hohen Gesundheitsausgaben liegt die Schweiz mit deutlichem Abstand an zweiter Stelle (Tabelle 1). Diesen Platz nimmt sie seit 1999 ein. Die genannten 25 Länder geben - wie auch alle 30 OECD-Mitglieder - für ihr Gesundheitssystem durchschnittlich 8,9\% ihres BIP aus. Die USA liegen $72 \%$ höher, die Schweiz 30\%.

Von 2000 bis 2004 stieg der Anteil am BIP im Schnitt der 25 Länder und auch in der Schweiz um $12 \%$. Die Tabelle 1 zeigt die extrem grossen Wachstumsunterschiede von nur 1\% (Griechenland) bis 37,9\% (Luxemburg). Diese Zahlen beruhen nicht nur auf der Zunahme der Kosten im Gesundheitswesen, sondern auch auf der Wirtschaftsentwicklung in der betreffenden

\section{Résumé}

L'aperçu sur la santé publique des pays de l'OCDE, intitulé «OECD Health Data - Statistics and Indicators for 30 Countries» [1] est mis à jour et publié chaque année en juin. L'édition de cette année (qui fête son $15^{\mathrm{e}}$ anniversaire) contient des données sur l'état de santé, les ressources, le recours aux soins, les dépenses de santé, le financement, la protection sociale, le marché des médicaments et les facteurs décisionnels non médicaux de l'état de santé. II contient aussi des indices démographiques et scientifiques. Cet aperçu représentatif comprend ainsi plus de $\mathbf{1 2 0 0}$ facteurs de référence au total.

En 2003 et en 2004, la Suisse a occupé le deuxième rang mondial en termes de dépenses de santé calculées en proportion du produit national brut. Elle est également restée au troisième rang pour les dépenses par tête et la part extrêmement élevée des personnes payant elles-mêmes les factures. La densité des hôpitaux se situe dans la moyenne tandis que celle des appareils médicaux est très élevée.

Periode, wie sie im Bruttoinlandsprodukt zum Ausdruck kommt.

\section{Ausgaben pro Kopf}

Der zweite klassische Kostenvergleich zwischen Ländern betrifft die Pro-Kopf-Ausgaben für die Gesundheit, gemessen in US-\$ (kaufkraftbereinigt, d.h. mit statistischer Berücksichtigung der unterschiedlichen Kaufkraft der einzelnen 
Länder). Die Schweiz ist in dieser Rangliste (Tabelle 2) für das Jahr 2004 wie schon 2003 auf dem dritten Platz zu finden, nach den USA und Luxemburg. Der Durchschnittswert der 25 Länder, für welche die Zahlen des Jahres 2004 vorliegen, ist 2582 US-\$. In dieser Statistik fällt die Höhe der US-Gesundheitsausgaben noch mehr auf: Sie sind 136\% höher als der Durchschnitt. Die Schweizer Ausgaben sind 58\% höher.

Die Zunahme der Pro-Kopf-Ausgaben zwischen 2000 und 2004 beträgt im Durchschnitt $32,7 \%$ bei den 25 Ländern, von denen die Zahlen für beide Jahre vorliegen. Die Zunahme ist in den USA fast gleich hoch, in der Schweiz etwas geringer.

Tabelle 1

Gesundheitsausgaben in Prozent des Bruttoinlandsprodukts BIP, 1990-2004.

\begin{tabular}{|c|c|c|c|c|}
\hline Land & 1990 & 2000 & 2004 & $\begin{array}{l}\text { Zunahme } 2000 \text { bis } 2004 \\
\text { in } \%\end{array}$ \\
\hline USA & 11.9 & 13.3 & 15.3 & 15.0 \\
\hline Schweiz [a] & 8.3 & 10.4 & 11.6 & 11.5 \\
\hline Deutschland [b] & 8.5 & 10.4 & 10.9 & 4.8 \\
\hline Frankreich [a] & 8.4 & 9.2 & 10.5 & 14.1 \\
\hline Island [a] & 7.9 & 9.2 & 10.2 & 10.9 \\
\hline Belgien [b] & 7.2 & 8.6 & 10.1 & 17.4 \\
\hline Portugal [a] & 6.2 & 9.4 & 10.0 & 6.4 \\
\hline Griechenland $[\mathrm{a}, \mathrm{c}]$ & 7.4 & 9.9 & 10.0 & 1.0 \\
\hline Kanada [a] & 9.0 & 8.9 & 9.9 & 11.2 \\
\hline Norwegen & 7.7 & 8.5 & 9.7 & 14.1 \\
\hline Österreich & 7.0 & 9.4 & 9.6 & 2.1 \\
\hline Niederlande [a] & 7.7 & 7.9 & 9.2 & 16.5 \\
\hline Australien [b] & 7.5 & 8.8 & 9.2 & 4.5 \\
\hline Schweden [a] & 8.3 & 8.4 & 9.1 & 8.3 \\
\hline Dänemark [a] & 8.3 & 8.3 & 8.9 & 7.2 \\
\hline Neuseeland & 6.9 & 7.7 & 8.4 & 9.1 \\
\hline Italien & 7.7 & 7.9 & 8.4 & 6.3 \\
\hline Ungarn [a] & 7.1 & 7.1 & 8.3 & 16.9 \\
\hline Grossbritannien & 6.0 & 7.3 & 8.3 & 13.7 \\
\hline Spanien [a] & 6.5 & 7.2 & 8.1 & 12.5 \\
\hline Luxemburg [a] & 5.4 & 5.8 & 8.0 & 37.9 \\
\hline Japan [b] & 5.9 & 7.6 & 8.0 & 5.3 \\
\hline Türkei & 3.6 & 6.6 & 7.7 & 16.7 \\
\hline Finnland & 7.8 & 6.7 & 7.5 & 11.9 \\
\hline Tschechische Republik [a] & 4.7 & 6.7 & 7.3 & 9.0 \\
\hline Irland & 6.1 & 6.3 & 7.1 & 12.7 \\
\hline Mexiko & 4.8 & 5.6 & 6.5 & 16.1 \\
\hline Polen & 4.9 & 5.7 & 6.5 & 14.0 \\
\hline Slowakische Republik [b] & $\ldots$ & 5.5 & 5.9 & 7.3 \\
\hline Südkorea & 4.4 & 4.8 & 5.6 & 16.7 \\
\hline Durchschnitt aller Länder & 7.0 & 8.0 & 8.9 & 11.7 \\
\hline
\end{tabular}

Die internationalen OECD-Vergleichszahlen zeigen einmal mehr die eklatanten Kostenunterschiede. Es gibt Länder mit anerkannt guten Gesundheitssystemen, die pro Kopf nur die Hälfte oder noch weniger ausgeben als Länder mit vergleichbaren Gesundheitssystemen. Dies bestätigt die alte Erkenntnis, dass die Qualität der Gesundheitsversorgung eines Landes von zahlreichen Faktoren abhängt. Die Höhe der Gesundheitsinvestitionen ist nur einer von vielen und nicht der entscheidende.

\section{Weltweit höchster Selbstzahleranteil}

Direkt aus der eigenen Tasche zahlten im Jahr 2004 die Schweizerinnen und Schweizer

Tabelle 2

Gesundheitsausgaben 2000 und 2004 in US-\$ (kaufkraftbereinigt) pro Kopf.

\begin{tabular}{|c|c|c|c|}
\hline Land & 2000 & 2004 & $\begin{array}{l}\text { Zunahme } \\
\text { in } \%\end{array}$ \\
\hline USA & 4588 & 6102 & 33.0 \\
\hline Luxemburg [b] & 2982 & 5089 & 70.7 \\
\hline Schweiz [b] & 3179 & 4077 & 28.2 \\
\hline Norwegen & 3080 & 3966 & 28.8 \\
\hline Island [b] & 2623 & 3331 & 27.0 \\
\hline Kanada [b] & 2503 & 3165 & 26.4 \\
\hline Frankreich [b] & 2450 & 3159 & 28.9 \\
\hline Österreich & 2667 & 3124 & 17.1 \\
\hline Belgien [c] & 2277 & 3044 & 33.7 \\
\hline Niederlande [b] & 2257 & 3041 & 34.7 \\
\hline Deutschland [c] & 2670 & 3005 & 12.5 \\
\hline Dänemark [b] & 2380 & 2881 & 21.1 \\
\hline Australien [c] & 2400 & 2876 & 19.8 \\
\hline Schweden [b] & 2271 & 2825 & 24.4 \\
\hline Irland & 1809 & 2596 & 43.5 \\
\hline Grossbritannien & 1858 & 2546 & 37.0 \\
\hline Italien & 2039 & 2392 & 17.3 \\
\hline Japan [c] & 1967 & 2249 & 14.3 \\
\hline Finnland & 1716 & 2235 & 30.2 \\
\hline Griechenland [a] & 1616 & 2162 & 33.8 \\
\hline Spanien [b] & 1520 & 2094 & 37.8 \\
\hline Neuseeland & 1605 & 2083 & 29.8 \\
\hline Portugal [b] & 1624 & 1813 & 11.6 \\
\hline Tschechische Republik [b] & 980 & 1361 & 38.9 \\
\hline Ungarn [b] & 856 & 1323 & 54.6 \\
\hline Südkorea & 778 & 1149 & 47.7 \\
\hline Polen & 590 & 805 & 36.4 \\
\hline Slowakische Republik [c] & 595 & 777 & 30.6 \\
\hline Mexiko & 506 & 662 & 30.8 \\
\hline Türkei & 451 & 580 & 28.6 \\
\hline Durchschnitt & 1961 & 2550 & 30.0 \\
\hline
\end{tabular}


Tabelle 3

Selbstzahlungen (Privathaushalte) in US-\$ (kaufkraftbereinigt) pro Kopf, 2000 und 2004.

\begin{tabular}{lcccc} 
Land & $\mathbf{2 0 0 0}$ & $\mathbf{2 0 0 4}$ & $\begin{array}{l}\text { DS 2004 } \\
=\text { Index 100 }\end{array}$ & $\begin{array}{l}\text { Zu-/Abnahme 2000 } \\
\text { bis 2004 }\end{array}$ \\
\hline Schweiz & 1045 & 1301 & 286.4 & 24.5 \\
\hline Griechenland & 726 & 977 & 215.1 & 34.6 \\
\hline USA & 683 & 803 & 176.8 & 17.6 \\
\hline Norwegen & 515 & 623 & 137.1 & 21.0 \\
\hline Island & 457 & 554 & 121.9 & 21.2 \\
\hline Spanien & 358 & 494 & 108.7 & 38.0 \\
\hline Kanada & 397 & 472 & 103.9 & 18.9 \\
\hline Italien & 465 & 468 & 103.0 & 0.6 \\
\hline Österreich & 445 & 459 & 101.0 & 3.1 \\
\hline Südkorea & 335 & 424 & 93.3 & 26.6 \\
\hline Finnland & 350 & 423 & 93.1 & 20.9 \\
\hline Dänemark & 380 & 400 & 88.0 & 5.3 \\
\hline Portugal & 360 & 391 & 86.1 & 8.6 \\
\hline Neuseeland & 247 & 359 & 79.0 & 45.3 \\
\hline Irland & 244 & 351 & 77.3 & 43.9 \\
\hline Luxemburg & 208 & 340 & 74.8 & 63.5 \\
\hline Mexiko & 258 & 335 & 73.7 & 29.8 \\
\hline Ungarn & 225 & 320 & 70.4 & 42.2 \\
\hline Frankreich & 257 & 239 & 52.6 & -7.0 \\
\hline Niederlande & 203 & 238 & 52.4 & 17.2 \\
\hline Polen & 177 & 226 & 49.7 & 27.7 \\
\hline Tschechische Republik & 93 & 141 & 31.0 & 51.6 \\
\hline Türkei & 125 & 112 & 24.7 & -10.4 \\
\hline Durchschnitt & 371.9 & 454.3 & 100.0 & 23.7 \\
\hline & & & & \\
\hline
\end{tabular}

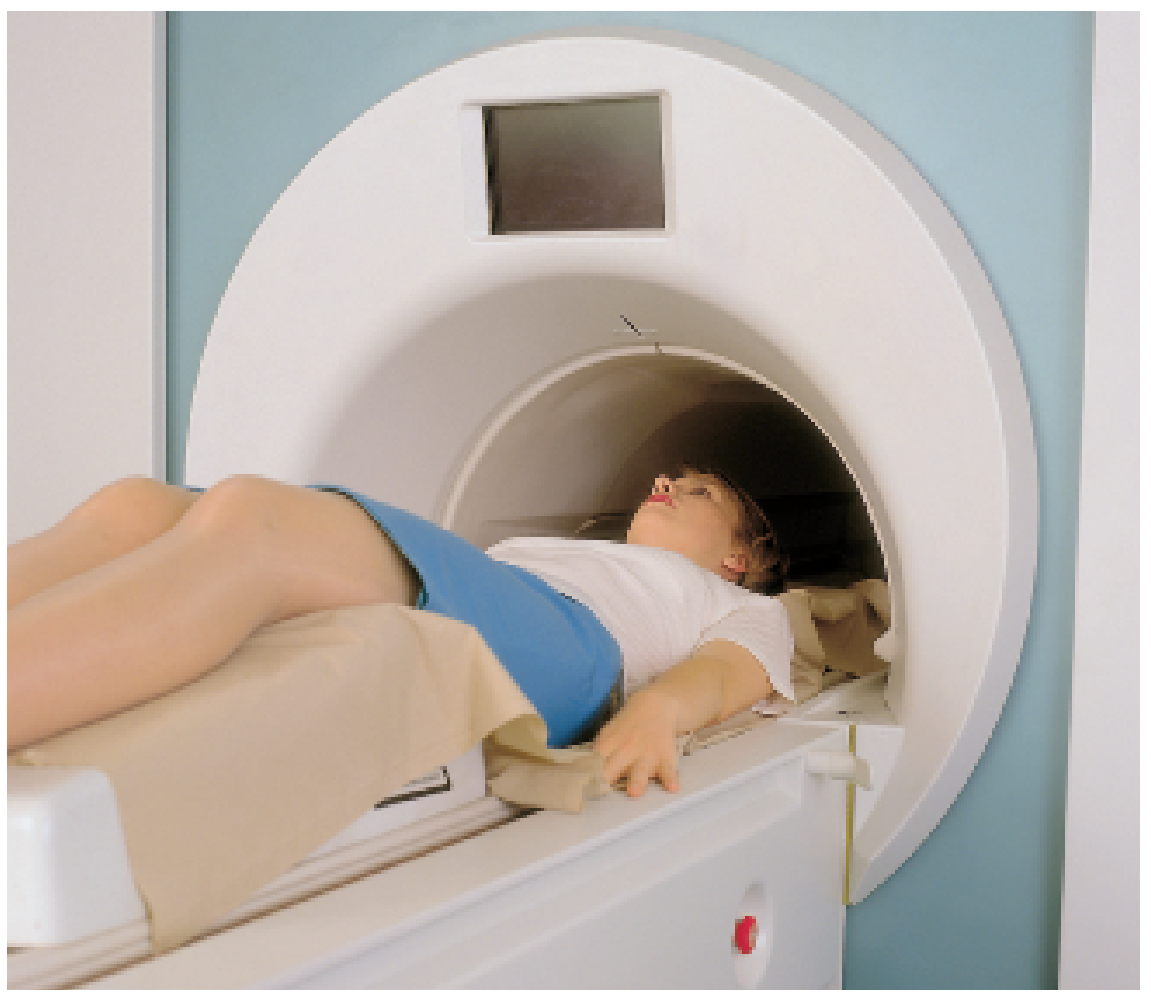

Die CT- und MRT-Dichte in der Schweiz ist im internationalen Vergleich überdurchschnittlich.
1301 US-\$ pro Kopf für das Gesundheitswesen, vor allem für rezeptfreie Medikamente, Zahnmedizin, Pflegeheime und Spitex (Tabelle 3). Diese Out-of-pocket-Zahlungen sind weitaus die höchsten aller 23 Länder, für welche die Zahlen des Jahres 2004 vorliegen. Der Durchschnitt dieser Länder beträgt 454 US-\$; die Schweizer zahlen mehr als zweieinhalbmal so viel (187\% mehr), die USA 77\% mehr. Gegenüber dem Vorjahr stiegen die Ausgaben der Selbstzahler in allen 23 Ländern im Durchschnitt um 6,1\%. Die Schweiz wird ihre weltweite Leaderposition 2005 und 2006 zweifellos noch ausbauen können, denn der politische Trend zur Direktbelastung der Haushalte und der Kranken nimmt immer noch zu.

Gemessen an den gesamten Gesundheitsausgaben beträgt der Selbstzahleranteil in der Schweiz 31,9\%, im Durchschnitt der 23 Länder beläuft er sich auf 20,5\%. Die Schweiz liegt also 56\% höher und mit den 31,9\% im dritten Rang nach Mexiko (50,6\%) und Griechenland $(45,2 \%)$.

Ein Vergleich der Jahre 2000 und 2004 ergibt folgendes Bild: die Ausgaben in kaufkraftbereinigten US-\$ stiegen im Schnitt um 23,7\% (Schweiz 24,5\%). Völlig anders war die Entwicklung beim Anteil der Selbstzahlungen an den nationalen Gesamt-Gesundheitsausgaben: er sank um 7,1\% (Rückgang in 18, Gleichstand in 2 und Wachstum in 3 Ländern).

\section{Tabelle 4}

Krankenhausbetten insgesamt, 2000 und 2004, je 1000 Einwohner.

\begin{tabular}{|c|c|c|c|}
\hline Land & 2000 & 2004 & $\begin{array}{l}\text { Zu- oder } \\
\text { Abnahme } \\
\text { in } \%\end{array}$ \\
\hline Japan & 14.7 & 14.2 & -3.4 \\
\hline Tschechische Republik & 8.8 & 8.7 & -1.1 \\
\hline Deutschland & 9.1 & 8.6 & -5.5 \\
\hline Ungarn & 8.1 & 7.8 & -3.7 \\
\hline Österreich & 8.6 & 7.7 & -10.5 \\
\hline Frankreich & 8.1 & 7.5 & -7.4 \\
\hline Südkorea & 6.1 & 7.3 & 19.7 \\
\hline Belgien & 7.1 & 6.8 & -4.2 \\
\hline Luxemburg & 6.9 & 6.7 & -2.9 \\
\hline Irland & 4.7 & 4.2 & -10.6 \\
\hline Grossbritannien & 4.3 & 4.1 & -4.7 \\
\hline Schweiz & 4.1 & 3.8 & -7.3 \\
\hline Norwegen & 3.8 & 3.7 & -2.6 \\
\hline Portugal & 3.8 & 3.7 & -2.6 \\
\hline USA & 3.5 & 3.3 & -5.7 \\
\hline Türkei & 2.6 & 2.6 & 0.0 \\
\hline Mexiko & 1.9 & 1.9 & 0.0 \\
\hline Durchschnitt & 6.2 & 6.0 & -3.1 \\
\hline
\end{tabular}




\section{Leicht rückläufige Zahl der Krankenhausbetten}

$\mathrm{Zu}$ den Betten pro 1000 Einwohner präsentiert die CD die Zahlen von 17 Ländern für 2004 (Tabelle 4). Die Variationen sind auch hier gross - von 1,9 in Mexiko über 3,8 in der Schweiz bis 14,2 in Japan. Gegenüber 2003 ist die Bettendichte in 9 Ländern gesunken (Schweiz: von 3,9 auf 3,8), in sechs Ländern gleichgeblieben und in zweien leicht gestiegen. Die Schweizer Bettendichte erscheint in dieser Statistik weniger exorbitant, als sie bei uns oft dargestellt wird: Die Schweiz ist im 10. Rang von 17 Ländern. Leider fehlen in dieser Statistik die Daten von 13 der 30 OECD-Mitgliedländer.

Der Vergleich 2004 mit 2000 für die 17 Länder ist eher überraschend: Zwar sank die Bettendichte in 14 Ländern, blieb in 2 Ländern gleich und stieg in einem Land. Im Durchschnitt dieser Staaten sank die Dichte aber nur von 6,2 auf 6,0 Betten.

\section{Medizintechnik: Ränge 2, 2, 3 und 5}

Seit jeher und seltsamerweise ist die Datenlage über medizintechnische Geräte fast in allen Ländern dürftig. Die hier besprochene CD bringt immerhin einige Einblicke (Tabelle 5), wenn auch leider die Daten zum Beispiel für die Niederlande, Norwegen, Schweden und die USA fehlen und für andere Länder nur teilweise vorhanden sind.

Für Computertomographen und Magnetresonanztomographen ist der Bestand für 2004 angegeben. Mit 17,9 CTs pro Million Einwohner ist die Schweiz im 5. Rang von 17 Ländern und damit etwas über dem Durchschnitt. Bei den MRTs belegt unser Land den 3. Rang nach Island und Österreich und ist 72\% über dem Länderdurchschnitt. Bezüglich Strahlentherapiegeräte und Lithotripter wird unser Land nur von Island bzw. Südkorea (Lithotripter) übertroffen. Unsere Werte sind 59 bzw. 88\% über dem Durchschnitt der 15 bzw. 13 Länder, für welche die 2004-Daten vorliegen.

Für einen Vergleich der Gerätedichte 2000 und 2004 sind je nach Gerät Daten von 12 bis 17 Ländern vorhanden. Die CT-Dichte stieg um 11\%, während die MRT-Dichte um 53\% zunahm. Die Zahl der Strahlentherapiegeräte pro Million Einwohner sank um 2\%, die Lithotripterdichte stieg um $18 \%$.

Tabelle 5

Medizintechnische Geräte pro Million Einwohner, 2000 und 2004.

\begin{tabular}{|c|c|c|c|c|c|c|c|c|}
\hline Land & $\begin{array}{l}\text { CT } \\
2000\end{array}$ & $\begin{array}{l}\text { CT } \\
2004\end{array}$ & $\begin{array}{l}\text { MRT } \\
2000\end{array}$ & $\begin{array}{l}\text { MRT } \\
2004\end{array}$ & $\begin{array}{l}\text { Str } \\
2000\end{array}$ & $\begin{array}{l}\text { Str } \\
2004\end{array}$ & $\begin{array}{l}\text { Lith } \\
2000\end{array}$ & $\begin{array}{l}\text { Lith } \\
2004\end{array}$ \\
\hline Australien & & & 3.5 & 3.7 & 5.2 & & 1.3 & \\
\hline Belgien & 21.8 & & 6 & & & & 4.8 & \\
\hline Dänemark & 11.4 & 14.6 & 5.4 & 10.2 & 5.4 & 6.3 & & \\
\hline Deutschland & 12.7 & 15.4 & 4.9 & 6.6 & 4.8 & 4.7 & 3 & 3.5 \\
\hline Finnland & 13.5 & 14.2 & 9.9 & 14 & 8.7 & 8.8 & 0.4 & 0.4 \\
\hline Frankreich & 9.5 & 7.5 & 2.6 & 3.2 & 6.1 & & 0.9 & 0.6 \\
\hline Grossbritannien [a] & 4.5 & 7 & 4.7 & 5 & & 3.9 & & \\
\hline Island & 21.3 & 17.1 & 10.7 & 17.1 & 14.2 & 13.7 & 3.6 & 3.4 \\
\hline Italien & 20.8 & 20.6 & 7.6 & 10.2 & 3.7 & 4.1 & & \\
\hline Kanada & & 10.8 & 2.5 & 4.9 & & & & 0.5 \\
\hline Luxemburg & 25.2 & 28.8 & 2.3 & 11.1 & 4.6 & 4.4 & 2.3 & 2.2 \\
\hline Mexiko & 1.4 & 3.1 & 0.6 & 1.7 & 1.1 & 1.4 & 0.4 & 1 \\
\hline Neuseeland & 8.8 & 12.1 & & & 9.9 & 7.6 & & \\
\hline Österreich & 25.8 & 28.5 & 10.9 & 14.9 & 4.2 & 4.6 & 1.7 & 1.8 \\
\hline Schweiz & 18.5 & 17.9 & 12.9 & 14.3 & 10.4 & 9.2 & 4.5 & 4.7 \\
\hline Spanien & 12 & 13.3 & 4.8 & 7.7 & 3.7 & 3.9 & 1.8 & 1.8 \\
\hline Südkorea & 28.4 & 31.5 & 5.4 & 11 & 5.3 & 4.6 & 4.4 & 7.8 \\
\hline Tschechische Republik & 9.6 & 12.6 & 1.7 & 2.8 & 6.3 & 7 & 2.8 & 3.1 \\
\hline Ungarn & 5.7 & 6.8 & 1.8 & 2.6 & 2.3 & 2.7 & 0.9 & 1.1 \\
\hline Durchschnitt & 14.8 & 15.4 & 5.5 & 8.3 & 6.0 & 5.8 & 2.3 & 2.5 \\
\hline Rang der Schweiz & 7 & 5 & 1 & 3 & 2 & 2 & 2 & 2 \\
\hline
\end{tabular}

